\title{
Optimal first-line chemotherapeutic treatment in patients with locally advanced or metastatic esophagogastric carcinoma: triplet versus doublet chemotherapy: a systematic literature review and meta-analysis
}

\author{
N. Haj Mohammad ${ }^{1}$ • E. ter $\operatorname{Veer}^{1}$ - L. Ngai $^{1}$ • R. Mali ${ }^{1}$ - M. G. H. van Oijen ${ }^{1}$ \\ H. W. M. van Laarhoven ${ }^{1}$
}

Published online: 13 August 2015

(C) The Author(s) 2015. This article is published with open access at Springerlink.com

\begin{abstract}
There is a debate whether triplet or doublet chemotherapy should be used as a first-line treatment in patients with advanced or metastatic esophagogastric cancer. Therefore, here we will review the available literature to assess the efficacy and safety of triplet versus doublet chemotherapy as a first-line treatment in patients with advanced esophagogastric cancer. We searched MEDLINE, Embase, and CENTRAL (Cochrane Central Register of Controlled Trials) between 1980 and March 2015 for randomized controlled phase II and III trials comparing triplet with doublet chemotherapy and abstracts of major oncology meetings from 1990 to 2014. Twenty-one studies with a total of 3475 participants were included in the meta-analysis for overall survival. An improvement in overall survival (OS) (hazard ratio (HR) 0.90, $95 \%$ confidence interval (CI) 0.83-0.97) and progression-free survival (PFS) (HR $0.80,95 \%$ CI $0.69-0.93)$ was observed in favor of
\end{abstract}

Electronic supplementary material The online version of this article (doi:10.1007/s10555-015-9576-y) contains supplementary material, which is available to authorized users.

N. Haj Mohammad

n.hajmohammad@amc.nl

1 Department of Medical Oncology, Academic Medical Center, F4-222 Meibergdreef 9, PO box 22600, 1100

DD Amsterdam, The Netherlands triplet. In addition, the use of triplet was associated with better objective response rate (ORR) (risk ratio 1.25, $95 \%$ CI 1.09-1.44) compared to doublet. The risks of grade 3-4 thrombocytopenia (6.2 vs $3.8 \%$ ), infection (10.2 vs $6.4 \%$ ), and mucositis (9.7 vs $4.7 \%$ ) were statistically significantly increased with triplet compared to doublet. This review shows that first-line triplet therapy is superior to doublet therapy in patients with advanced esophagogastric cancer. However, the survival benefit is limited and the risks of grade 3-4 thrombocytopenia, infection, and mucositis are increased.

Keywords First-line treatment - Triplet chemotherapy · Doublet chemotherapy $\cdot$ Palliative chemotherapy $\cdot$ Esophageal cancer $\cdot$ Gastric cancer

\section{Introduction}

Gastric and esophageal cancers are respectively the second and the sixth most common cause of cancer-related deaths worldwide. The only potentially curative option involves resection. Unfortunately, the majority of patients presents with advanced disease or develops metastases after treatment with curative intent. In these patients, palliative systemic chemotherapy improves survival and quality of life, compared to best supportive care $[1-3]$.

Combination therapies have been associated with substantially higher response rates and survival compared to monotherapy [4, 5]. However, the optimal regimen for first-line palliative chemotherapy has yet to be clearly established and the question whether a three-drug 
Fig. 1 PRISMA flow diagram of literature search and study selection

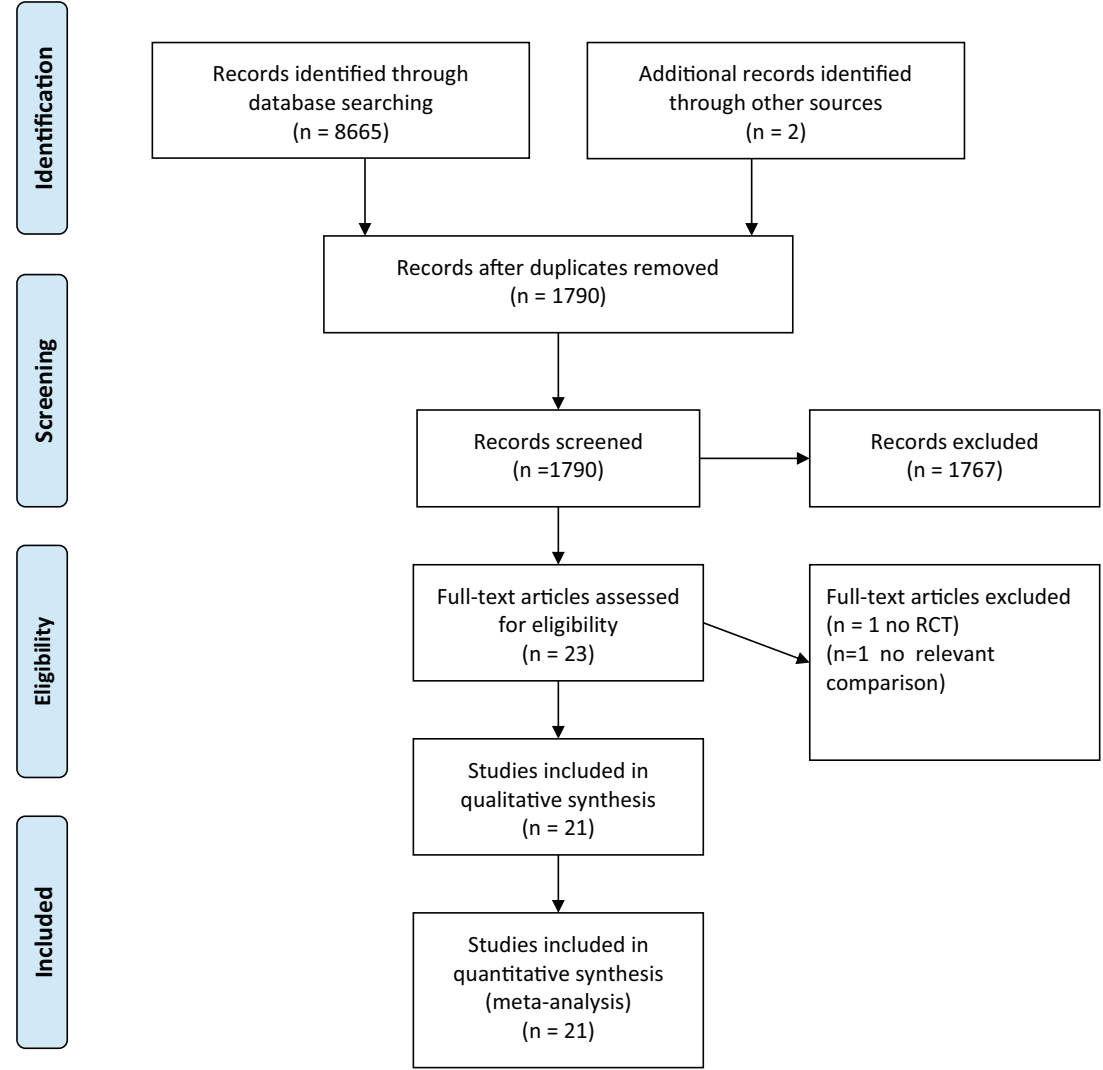

regimen is more effective than a potentially less toxic doublet is a point of debate. A Cochrane review published in 2010 concluded that "two and three-drug regimens including 5-FU, cisplatin, with or without an anthracycline are reasonable treatment options [6]." This ambiguity is reflected in various guidelines. According to the National Comprehensive Cancer Network (NCCN) guidelines of 2015 two-drug regimens are preferred and three-drug cytotoxic regimens should be reserved for medically fit patients with good performance scores and access to frequent toxicity evaluation [7]. The European Society for Medical Oncology (ESMO) guidelines of 2013 state that "combination regimens incorporating a platinum agent and a fluoropyrimidine are generally used. It remains controversial whether a triplet regimen is needed [8]." In recently published randomized trials introducing targeted therapies in first-line treatment, mainly doublets have been used as the backbone chemotherapy [9-11], although one trial used a triplet [12].

Therefore, here we will systematically review the existing literature on triplet or doublet therapy in terms of overall survival, progression-free survival, objective response rate, and safety in the management of advanced esophagogastric cancer.

\section{Methods}

\subsection{Search methods}

A search was conducted at the Cochrane Central Register of Controlled Trials (CENTRAL), MEDLINE, and EMBASE up to March 2015. The search strategy contained medical subject headings (MESH) and text words for esophageal and gastric cancer and all established chemotherapy compounds in esophageal and gastric cancer. We searched all abstracts from the American Society of Clinical Oncology (ASCO) and the ESMO conferences held between 1990 and 2014. The research question was registered in PROSPERO in September 2014 (registration: CRD42014014480).

\subsection{Study selection}

Randomized phased II or III studies were included. We included studies in abstract form only if information on study design, characteristics of participants, interventions, and outcomes was available in English. We excluded crossover studies and quasi randomized studies. Patients had advanced, recurrent, or metastatic 
Table 1 Characteristics of the included studies

\begin{tabular}{|c|c|c|c|c|c|c|c|c|c|c|c|c|c|c|c|c|}
\hline \multirow[t]{3}{*}{ Study } & \multirow[t]{3}{*}{ Number } & \multirow[t]{3}{*}{ Arms } & \multicolumn{2}{|c|}{ Efficacy } & \multicolumn{2}{|l|}{ Age } & \multirow{2}{*}{\multicolumn{2}{|c|}{$\frac{\text { Sex }}{\text { Male }}$}} & \multicolumn{4}{|c|}{ Disease status } & \multicolumn{4}{|c|}{ ECOG } \\
\hline & & & \multirow{2}{*}{$\begin{array}{l}\text { OS } \\
\text { Median } \\
\text { months }\end{array}$} & \multirow[t]{2}{*}{ PFS } & \multirow[t]{2}{*}{ Median } & \multirow[t]{2}{*}{ Range } & & & \multicolumn{2}{|l|}{ LA } & \multicolumn{2}{|l|}{ ME } & \multicolumn{2}{|l|}{$0-1$} & \multicolumn{2}{|l|}{$\geq 2$} \\
\hline & & & & & & & $N$ & $\%$ & $N$ & $\%$ & $N$ & $\%$ & $N$ & $\%$ & $N$ & $\%$ \\
\hline \multirow[t]{2}{*}{ Ajani 2005 [16] } & 79 & $\mathrm{DTX}+\mathrm{C}$ is $+\mathrm{FU}$ & 9.6 & NA & 57 & $21-83$ & 53 & 70 & 4 & 6 & 72 & 95 & $79 *$ & 100 & 0 & 0 \\
\hline & 76 & $\mathrm{DTX}+\mathrm{C}$ is & 10.5 & NA & 57 & $30-76$ & 61 & 77 & 1 & 1 & 75 & 95 & 75 & 99 & 1 & 1 \\
\hline Al-Batran 2013 [17] & 72 & $\mathrm{DTX}+\mathrm{Ox}+\mathrm{FU}+\mathrm{LV}$ & 17.3 & 9.1 & 69 & $65-81$ & 51 & 71 & 22 & 31 & 50 & 69 & 67 & 93 & 5 & 7 \\
\hline & 71 & $\mathrm{Ox}+\mathrm{FU}+\mathrm{LV}$ & 14.4 & 6.7 & 70 & $65-82$ & 45 & 63 & 22 & 32 & 49 & 68 & 65 & 92 & 6 & 9 \\
\hline Cullinan1985 [35] & 51 & $\mathrm{FU}+\mathrm{Doxo}+\mathrm{MMC}$ & NA & NA & $60^{\#}$ & NA & 39 & 76 & 20 & 39 & 31 & 61 & 32 & 63 & 19 & 37 \\
\hline & 49 & $\mathrm{Doxo}+\mathrm{FU}$ & NA & NA & $63^{\#}$ & NA & 37 & 76 & 18 & 37 & 31 & 63 & 33 & 67 & 16 & 33 \\
\hline Douglass 1984 [36] & 39 & $\mathrm{FU}+\mathrm{Doxo}+\mathrm{Me}$ & 24.5 & NA & 59.5 & $43-76$ & 28 & 71 & 0 & 0 & 39 & 100 & 30 & 77 & 9 & 23 \\
\hline & 46 & $\mathrm{FU}+\mathrm{Doxo}+\mathrm{MMC}$ & 29.5 & NA & 61.0 & $32-81$ & 35 & 76 & 0 & 0 & 46 & 100 & 30 & 65 & 16 & 35 \\
\hline & 48 & $\mathrm{FU}+\mathrm{Me}$ & 13.5 & NA & 62.0 & $24-79$ & 38 & 80 & 0 & 0 & 48 & 100 & 35 & 72 & 13 & 28 \\
\hline & 46 & Doxo+MMC & 19.0 & NA & 58.0 & $33-78$ & 37 & 80 & 0 & 0 & 46 & 100 & 28 & 61 & 18 & 39 \\
\hline Guimbaud 2014 [18] & 209 & Epi + Cis + Cape & 9.5 & 5.3 & 61 & $28-84$ & 154 & 74 & 36 & 17 & 173 & 83 & 169 & 81 & 36 & 17 \\
\hline & 207 & FU+Iri & 9.7 & 5.7 & 61 & $29-81$ & 155 & 75 & 31 & 15 & 176 & 85 & 173 & 84 & 27 & 13 \\
\hline Kim 1993 [37] & 110 & $\mathrm{FU}+\mathrm{Doxo}+\mathrm{MMC}$ & 6.84 & NA & 54 & $19-77$ & 68 & 62 & NA & NA & NA & NA & 75 & 68 & 23 & 21 \\
\hline & 112 & $\mathrm{Cis}+\mathrm{FU}$ & 8.61 & NA & 51 & $20-68$ & 71 & 63 & NA & NA & NA & NA & 83 & 74 & 20 & 18 \\
\hline Kim 2001 [38] & 60 & $\mathrm{Epi}+\mathrm{Cis}+\mathrm{FU}$ & 8.5 & NA & 55 & NA & 45 & 75 & 3 & 5 & 57 & 95 & 54 & 90 & 6 & 10 \\
\hline & 60 & $\mathrm{Cis}+\mathrm{FU}$ & 7.3 & NA & 56 & NA & 42 & 70 & 3 & 5 & 57 & 95 & 53 & 88 & 7 & 12 \\
\hline Koizumi 2004 [39] & 33 & 5-DFUR + Cis + MMC & 8.03 & NA & 58 & $36-79$ & 19 & 58 & NA & NA & NA & NA & 16 & 48 & 13 & 39 \\
\hline & 29 & 5-DFUR + Cis & 5.97 & NA & 58 & $37-79$ & 17 & 59 & NA & NA & NA & NA & 25 & 86 & 6 & 24 \\
\hline KRCCG 1992 [40] & 25 & $\mathrm{Epi}+\mathrm{Cis}+\mathrm{FU}$ & 6.9 & NA & NA & NA & NA & NA & NA & NA & NA & NA & NA & NA & NA & NA \\
\hline & 22 & $\mathrm{Cis}+\mathrm{FU}$ & 4.0 & NA & NA & NA & NA & NA & NA & NA & NA & NA & NA & NA & NA & NA \\
\hline Li 2011 [41] & 50 & $\mathrm{PTX}+\mathrm{C}$ is $+\mathrm{FU}$ & 10.6 & NA & 59 & $20-74$ & 32 & 68 & 22 & 46 & 28 & 56 & NA & NA & NA & NA \\
\hline & 44 & $\mathrm{Ox}+\mathrm{FU}$ & 9.9 & NA & 58 & $20-75$ & 31 & 70 & 17 & 41 & 27 & 61 & NA & NA & NA & NA \\
\hline Lin 2009 [42] & 13 & $\mathrm{FU}+\mathrm{Ox}+\mathrm{PTX}$ & NA & NA & 55 & $36-67$ & 18 & 72 & NA & NA & NA & NA & NA & NA & NA & NA \\
\hline & 12 & FU+Iri & NA & NA & 55 & $36-67$ & 18 & 72 & NA & NA & NA & NA & NA & NA & NA & NA \\
\hline Maiello 2011 [43] & 36 & Epi + Cis + Cap & NA & NA & 58 & $39-74$ & 22 & 60 & NA & NA & NA & NA & NA & NA & NA & NA \\
\hline & 31 & $\mathrm{DTX}+\mathrm{FU}$ & NA & NA & 61 & $44-75$ & 23 & 74 & NA & NA & NA & NA & NA & NA & NA & NA \\
\hline Park 2008 [19] & 45 & $\mathrm{Cis}+\mathrm{Iri}+\mathrm{FU}$ & 10.5 & 6.2 & 52 & $29-70$ & 30 & 67 & 0 & 0 & 45 & 100 & 38 & 84 & 7 & 16 \\
\hline & 46 & $\mathrm{Iri}+\mathrm{FU}$ & 10.7 & 4.8 & 55 & $26-73$ & 30 & 67 & 0 & 0 & 45 & 100 & 35 & 78 & 11 & 29 \\
\hline Roth 1999 [44] & 61 & $\mathrm{Epi}+\mathrm{Cis}+\mathrm{FU}$ & 9.6 & NA & 54 & NA & 37 & 61 & 12 & 22 & 42 & 78 & 24 & 39 & 30 & 61 \\
\hline & 61 & Epi+FU & 7.1 & NA & 56 & NA & 42 & 69 & 16 & 30 & 40 & 84 & 27 & 44 & 29 & 56 \\
\hline Roth 2007 [20] & 40 & $\mathrm{Epi}+\mathrm{Cis}+\mathrm{FU}$ & 8.3 & NA & 59 & $32-71$ & 30 & 75 & 7 & 17 & 33 & 83 & 40 & 100 & 0 & 0 \\
\hline & 38 & $\mathrm{DTX}+\mathrm{C}$ is & 11.0 & NA & 58 & $40-70$ & 29 & 76 & 7 & 18 & 31 & 82 & 38 & 100 & 0 & 0 \\
\hline & 41 & $\mathrm{DTX}+\mathrm{Cis}+\mathrm{FU}$ & 10.4 & NA & 61 & $35-78$ & 30 & 73 & 2 & 5 & 39 & 95 & 41 & 100 & 0 & 0 \\
\hline Thuss-Patience 2005 [45] & 45 & $\mathrm{Epi}+\mathrm{Cis}+\mathrm{FU}$ & 9.7 & NA & 63 & $33-75$ & 36 & 80 & 1 & 2 & 44 & 98 & 44 & 98 & 1 & 2 \\
\hline & 45 & $\mathrm{DTX}+\mathrm{FU}$ & 9.5 & NA & 62 & $34-75$ & 29 & 64 & 1 & 2 & 44 & 98 & 42 & 95 & 2 & 4 \\
\hline Van Cutsem 2006 [22] & 227 & $\mathrm{DTX}+\mathrm{Cis}+\mathrm{FU}$ & 9.2 & NA & 55 & $26-79$ & 159 & 72 & 6 & 3 & 213 & 96 & 218 & 99 & 3 & 1 \\
\hline & 230 & $\mathrm{Cis}+\mathrm{FU}$ & 8.6 & NA & 55 & $25-76$ & 158 & 71 & 6 & 3 & 217 & 97 & 221 & 99 & 3 & 1 \\
\hline Van Cutsem 2015 [21] & 89 & $\mathrm{DTX}+\mathrm{Ox}+\mathrm{FU}$ & 14.6 & 7.6 & 58 & NA & 61 & 69 & 0 & 0 & 89 & 100 & 87 & 98 & 2 & 2 \\
\hline & 86 & $\mathrm{DTX}+\mathrm{Ox}+\mathrm{Cap}$ & 11.3 & 5.6 & 59 & NA & 64 & 74 & 0 & 0 & 86 & 100 & 83 & 97 & 3 & 3 \\
\hline & 79 & $\mathrm{DTX}+\mathrm{Ox}$ & 9.0 & 4.5 & 59 & NA & 51 & 65 & 0 & 0 & 79 & 100 & 77 & 99 & 1 & 1 \\
\hline Van Hoefer 2000 [23] & 133 & FU+Doxo+MTX & 6.7 & 3.3 & 58 & $30-74$ & 96 & 72 & 22 & 17 & 111 & 83 & 117 & 88 & 16 & 12 \\
\hline & 134 & $\mathrm{Cis}+\mathrm{FU}$ & 7.2 & 4.1 & 57 & $24-74$ & 91 & 68 & 21 & 16 & 113 & 84 & 114 & 85 & 20 & 15 \\
\hline & 132 & Eto $+F U+L V$ & 7.2 & 3.3 & 59 & $25-74$ & 90 & 68 & 22 & 17 & 110 & 83 & 120 & 91 & 12 & 9 \\
\hline Wang 2015 [46] & 119 & $\mathrm{DTX}+\mathrm{Cis}+\mathrm{FU}$ & 10.2 & 7.2 & 57 & $19-80$ & 81 & 68 & 30 & 25 & 89 & 75 & 115 & 97 & 4 & 3 \\
\hline & 115 & $\mathrm{Cis}+\mathrm{FU}$ & 8.5 & 4.9 & 56 & $33-74$ & 88 & 77 & 26 & 23 & 89 & 77 & 108 & 94 & 7 & 6 \\
\hline
\end{tabular}


Table 1 (continued)

\begin{tabular}{|c|c|c|c|c|c|c|c|c|c|c|c|c|c|c|c|c|}
\hline \multirow[t]{3}{*}{ Study } & \multirow[t]{3}{*}{ Number } & \multirow[t]{3}{*}{ Arms } & \multicolumn{2}{|c|}{ Efficacy } & \multicolumn{2}{|l|}{ Age } & \multirow{2}{*}{\multicolumn{2}{|c|}{$\frac{\text { Sex }}{\text { Male }}$}} & \multicolumn{4}{|c|}{ Disease status } & \multicolumn{4}{|c|}{ ECOG } \\
\hline & & & \multirow{2}{*}{$\begin{array}{l}\text { OS } \\
\text { Mediar } \\
\text { month }\end{array}$} & \multirow{2}{*}{ PFS } & \multirow[t]{2}{*}{ Median } & \multirow[t]{2}{*}{ Range } & & & \multicolumn{2}{|l|}{ LA } & \multicolumn{2}{|l|}{ ME } & \multicolumn{2}{|c|}{$0-1$} & \multicolumn{2}{|c|}{$\geq 2$} \\
\hline & & & & & & & $N$ & $\%$ & $N$ & $\%$ & $N$ & $\%$ & $N$ & $\%$ & $N$ & $\%$ \\
\hline \multirow[t]{2}{*}{ Yun 2010 [47] } & 44 & Epi + Cis + Cap & 13.8 & 6.5 & 55 & $35-71$ & 28 & 64 & NA & NA & NA & NA & 40 & 91 & 1 & 9 \\
\hline & 47 & $\mathrm{Cis}+\mathrm{Cap}$ & 12.7 & 6.4 & 58 & $33-75$ & 34 & 72 & NA & NA & NA & NA & 41 & 87 & 4 & 13 \\
\hline
\end{tabular}

OS overall survival, $P F S$ progression-free survival, TTP time to progression, $L A$ locally advanced, $M E$ metastatic disease, ECOG Eastern Cooperative Oncology Group performance status, NA not applicable, DTX docetaxel, PTX palictaxel, Cis cisplatin, FU fluorouracil, Cap capecitabine, 5-DFUR doxifluridine, $O x$ oxaliplatin, Doxo doxorubicin, Epi epirubicin, Iri irinotecan, MTX methotrexate, MMC mitomycin C, Eto etoposide

*Karnofksi 80-100

\# mean age OR

adenocarcinoma of the distal esophagus, gastroesophageal junction, or stomach. They were not previously treated with chemotherapy (or $\geq 6$ months ago in adjuvant setting). Treatment was defined as

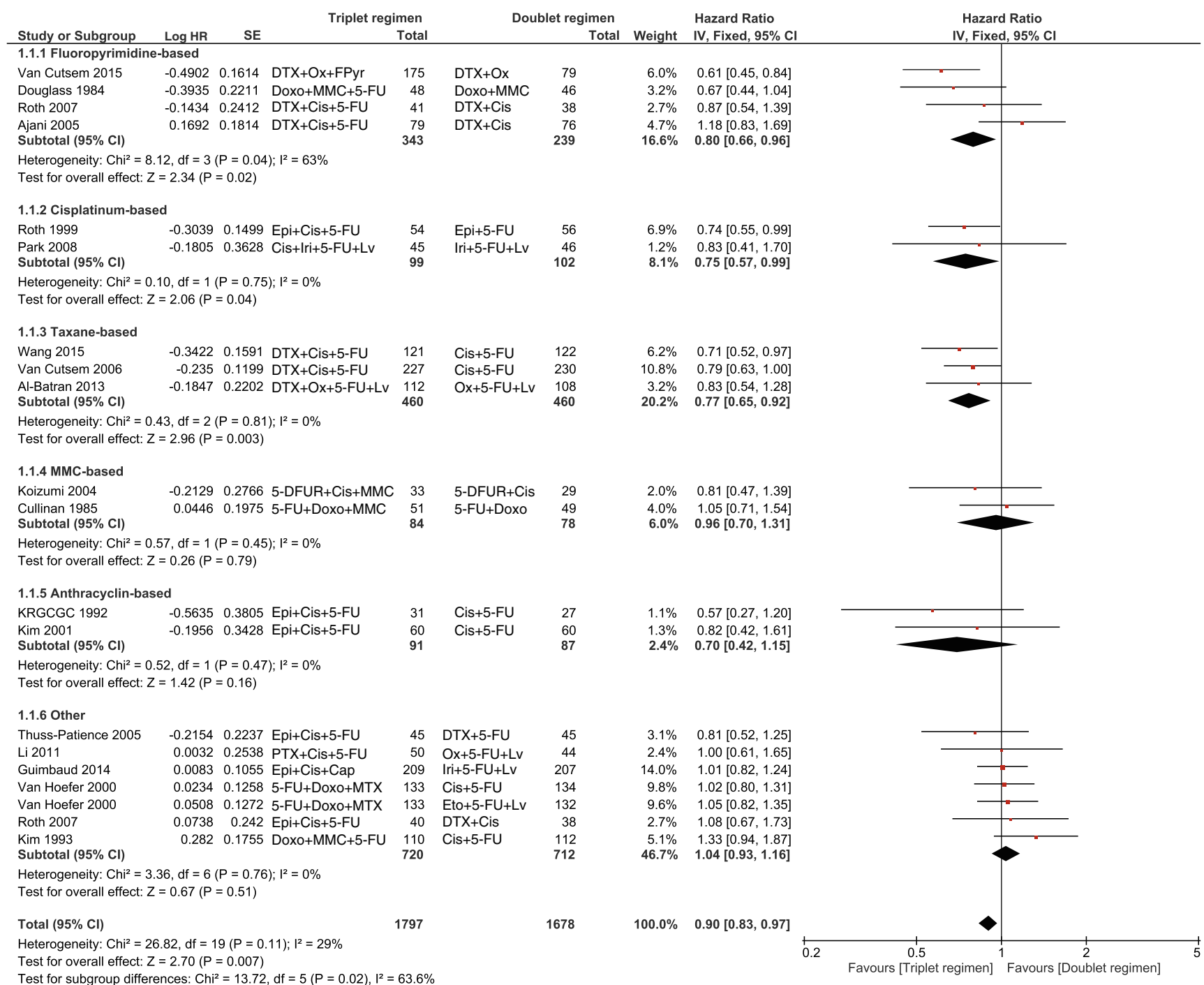

Fig. 2 When examining the subgroups, taxane and cisplatin showed a significant benefit 


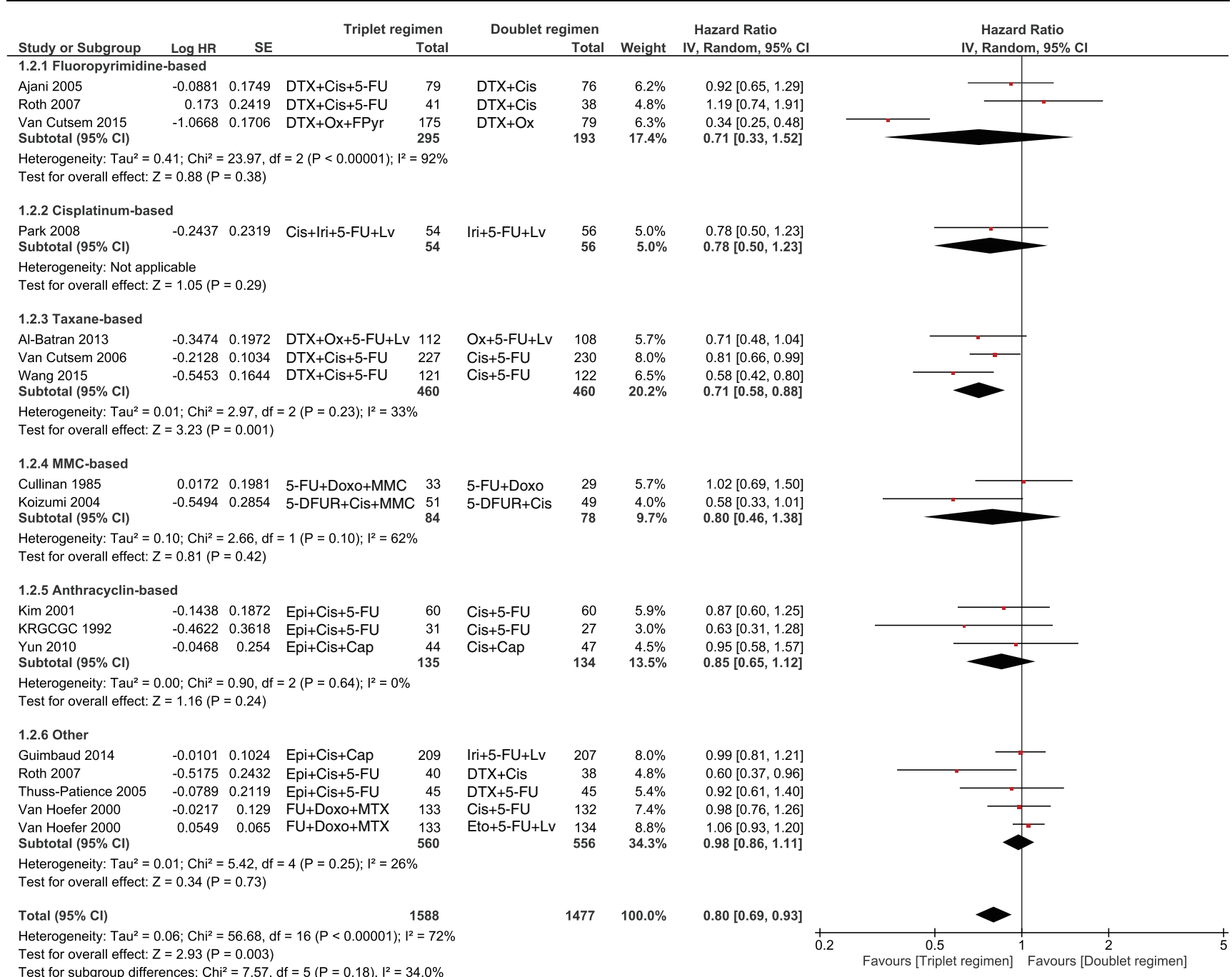

Fig. 3 A significant benefit was observed for PFS in favor of a triplet, which was mainly based on the addition of a taxane to the doublet

intravenous or oral chemotherapy and we excluded targeted therapy/biological therapy. Subgroups were made and named after the third compound that was added to the identical backbone in both arms. One subgroup "other" was created that contained a triplet and a doublet without the presence of an identical doublet backbone.

\subsection{Data extraction}

NHM, MA, and EV conducted the search. NHM and EV independently scrutinized titles and abstracts and if applicable the full articles. HvL decided in case of disagreement between NHM and EV. NHM, RM, and EV extracted the study characteristics and outcome data. The primary outcome was overall survival (OS). Overall survival was defined as the time between date of randomization and date of death or last date of follow-up.

Secondary outcomes were progression-free survival (PFS), objective response rate (ORR), and toxicity. Treatment-related toxicity was defined as the highest grade of toxicity per participant. Toxicity data, when available, were recorded if scored as grade 3-4 toxicity.

\subsection{Assessment of risk of bias}

All selected studies were critically appraised using an assessment form designed for the topic of this review according to the Cochrane Handbook for Systematic Reviews of Interventions 4.2.2 [13]. Risk of bias caused by the absence of blinded review of CT scans was not scored as high risk, since our primary outcome OS would not be influenced by this parameter. NHM and 


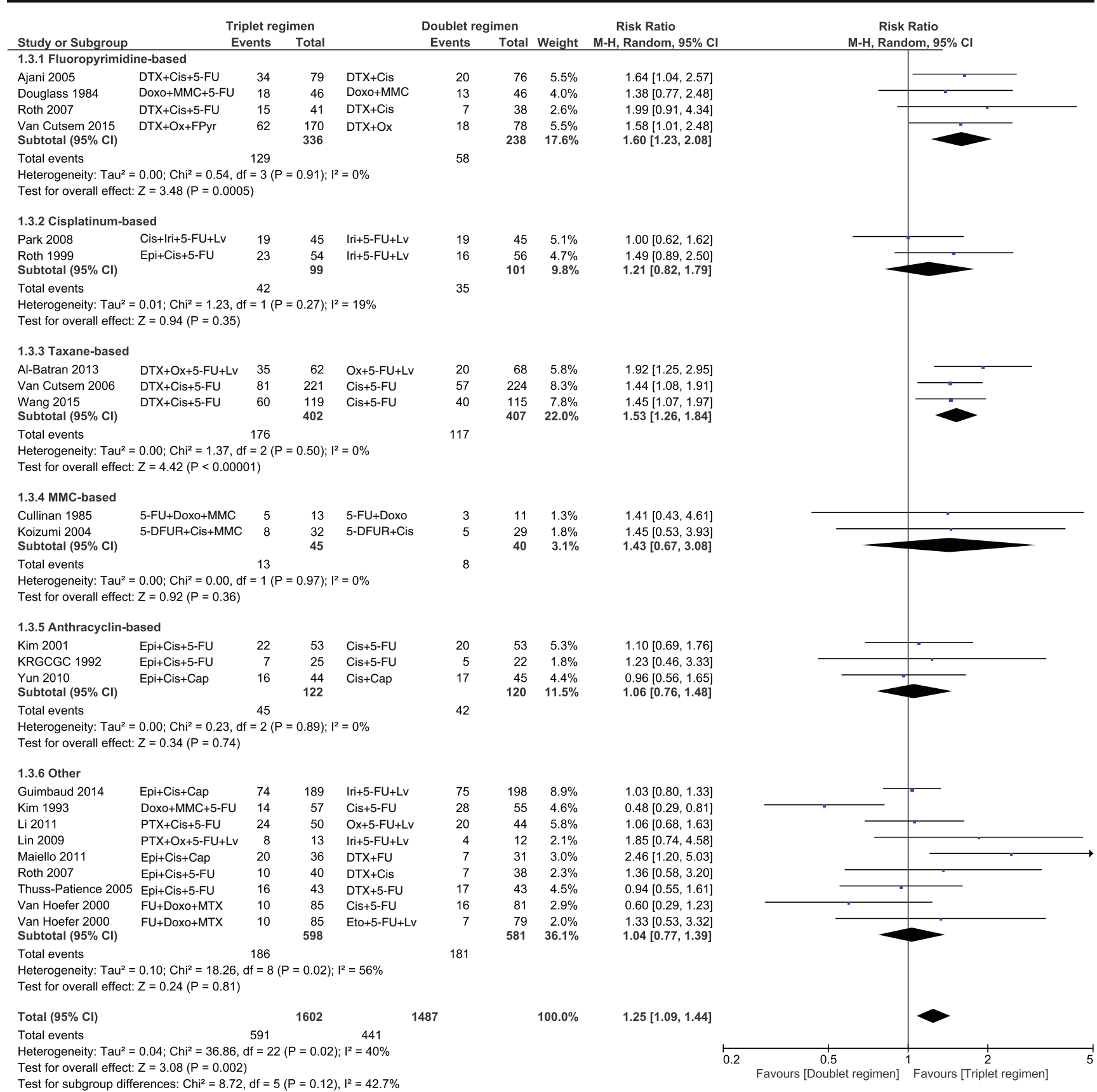

Fig. 4 The use of a triplet was associated with a better ORR compared to a doublet, which was mainly due to triplets with a fluoropyrimidine or taxane

EV assessed the risk of bias. HvL decided in case of disagreement. If data were missing, we contacted the first author to obtain further information.

\subsection{Statistical analysis}

Survival analysis was conducted using the intention-totreat population. A fixed model was used to calculate the pooled hazard ratio (HR) estimate. HRs for mortality were combined using an inverse variance method based on a logarithmic conversion; $95 \%$ confidence intervals were used to determine the standard error using according to Tierney et al. [14]. The traditional $Q$ test and the $I^{2}$ statistic were used to evaluate heterogeneity [15]. Where heterogeneity levels were moderate or high (defined as $I^{2} \geq 50 \%$ ), we used a random effects model. We repeated the primary analysis and investigated the influence of risk of bias, continent of conduction of the trial, and studies that compared triplet 


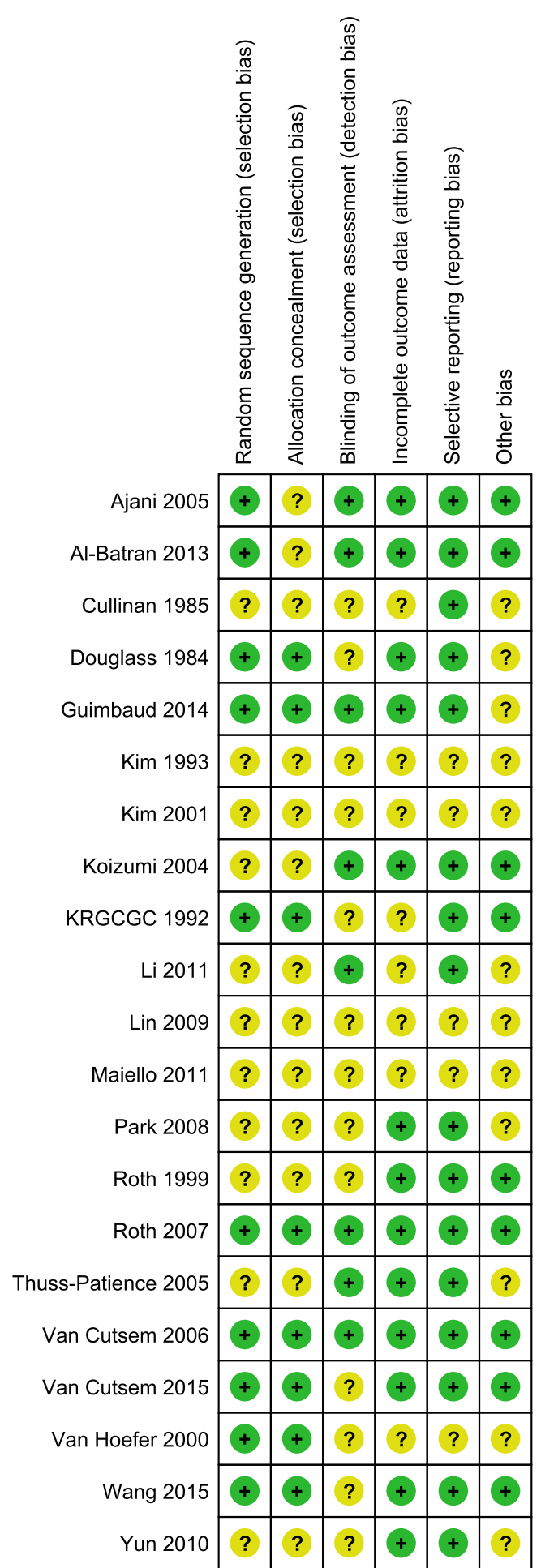

Fig. 5 Risk of bias assessment

versus doublet without identical backbones, excluding those trials with a high risk of bias score, trials conducted in Asia, and trials of which the backbone was not identical. All meta-analyses were performed with Cochrane Review Manager, version 5.3.

\section{Study outcomes}

\subsection{Description of studies}

We identified 6715 articles from the database search. After duplication, 1490 articles were screened on title and abstract. Of these papers, 1467 were excluded: no randomized controlled trials, reviews, and no comparison of a doublet versus a triplet. Twenty-three articles were scrutinized as full text. Finally, 21 randomized controlled studies were included in the qualitative and quantitative analysis (Fig. 1.)

Twenty-two studies with in total 3475 participants investigating a triplet versus a doublet were included. Table 1 shows the characteristics of the studies included in the meta- analysis.

\subsection{Overall survival, progression-free survival, and objective response rate}

A significant improvement in OS with a low heterogeneity was observed in favor of a triplet (HR 0.90, $95 \%$ confidence interval (CI) $0.83-0.97, I^{2}=29 \%$ ). When examining the subgroups, especially the triplets with fluoropyrimidine, taxane and cisplatin showed a significant benefit (Fig. 2.). Also, a significant benefit was observed for PFS in favor of a triplet (HR 0.80, $95 \%$ CI 0.69-0.93; Fig. 3), which was mainly based on the addition of a taxane to the doublet.

In addition, the use of a triplet was associated with a better ORR compared to a doublet (risk ratio $=1.25,95 \%$ CI 1.09 1.44). This was mainly due to triplets with a fluoropyrimidine or taxane (Fig. 4.)

\subsection{Risk of bias and sensitivity analyses}

Data on outcome measures and risk of bias were requested from all authors, but unfortunately only $28 \%$ of the corresponding authors provided further information. Risk of bias assessments are shown in supplementary table 1 (Fig. 5).

A sensitivity analysis excluding those trials that were conducted in Asia had no impact on the HR (0.90, $95 \%$ CI $0.93-0.97$ ) for OS (Fig. 6). Sensitivity analysis excluding trials on the basis of low quality was not possible due to the lack of data. Instead, studies with "unknown" risk of bias on "random sequence" and "allocation concealment" were excluded in a sensitivity analysis that showed a comparable HR of $0.92(95 \%$ CI 0.84-1.02) (Fig. 7). Sensitivity analysis excluding those trials that compared a triplet versus a doublet without the presence of two identical compounds in 


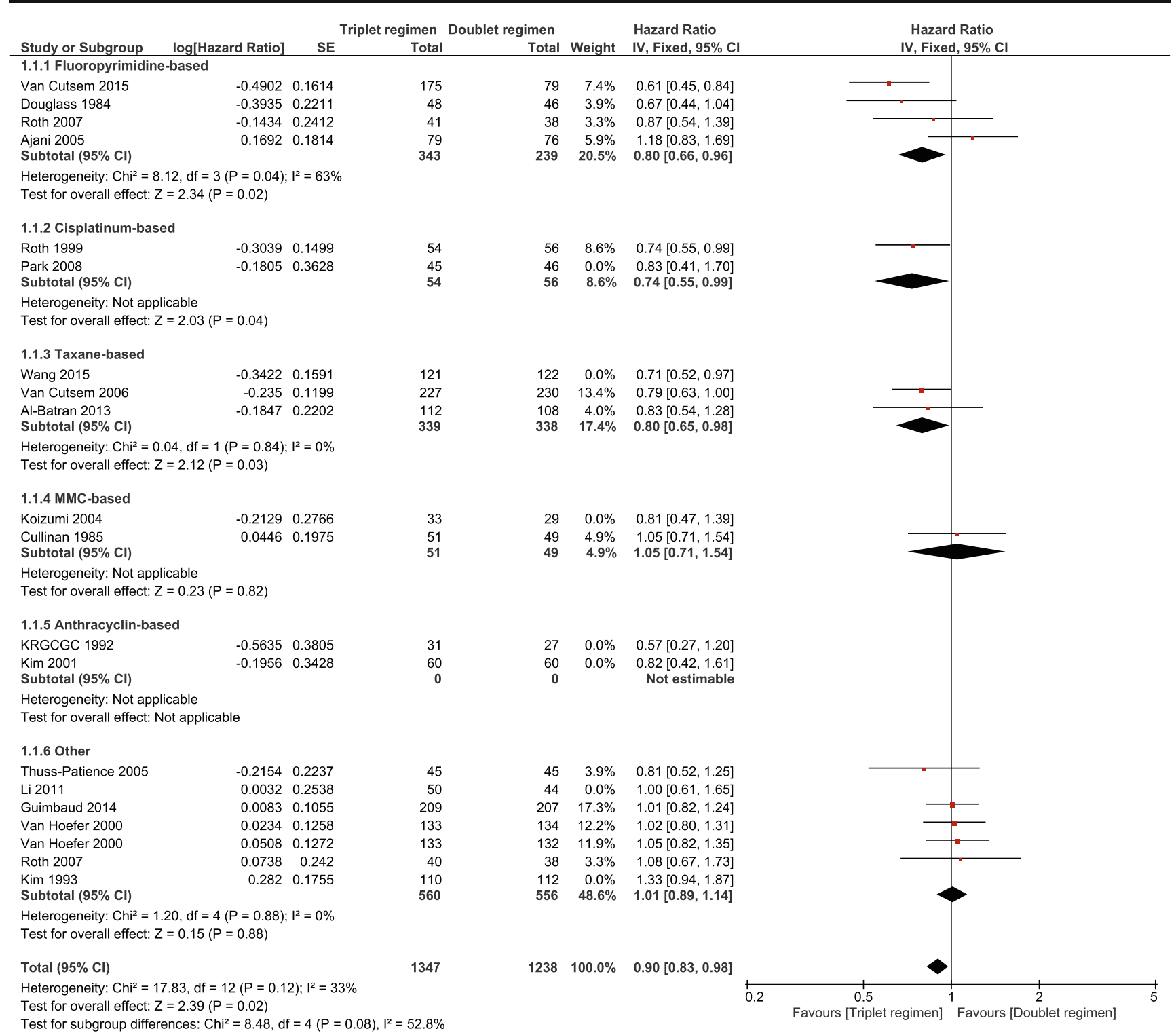

Fig. 6 Sensitivity analysis excluding those trials that were conducted in Asia

both arms showed a lower HR for OS of 0.79 (95\% CI 0.71-0.88 (Fig. 8)).

\subsection{Toxicity}

The risk of grade 3-4 thrombocytopenia (6.2 vs $3.7 \%$ ), infection (10.2 vs $6.4 \%$ ), and mucositis (9.7 vs $4.7 \%$ ) was significantly increased with a triplet compared to a doublet (Table 2).

\section{Discussion}

Our systematic review and meta-analysis showed that a triplet regimen was superior to a doublet regimen in terms of overall survival, progression-free survival, and objective response rate. However, hazard ratios were of limited clinical relevance and toxicity grades 3 and 4 were significantly higher in the triplet regimens.

The positive effects on OS of adding a fluoropyrimidine or a taxane to a doublet were based on a relatively large number of patients ( $>500$ patients), making the findings in these subgroups very robust. The favorable outcome of a triplet with cisplatin over a doublet was based on a smaller number of patients. Our results are in line with the previously published findings by Wagner et al. [6] and are considered clinically relevant given the widespread use of fluoropyrimidine, taxane, and cisplatin containing triplets in routine practice in Europe [16-23]. 


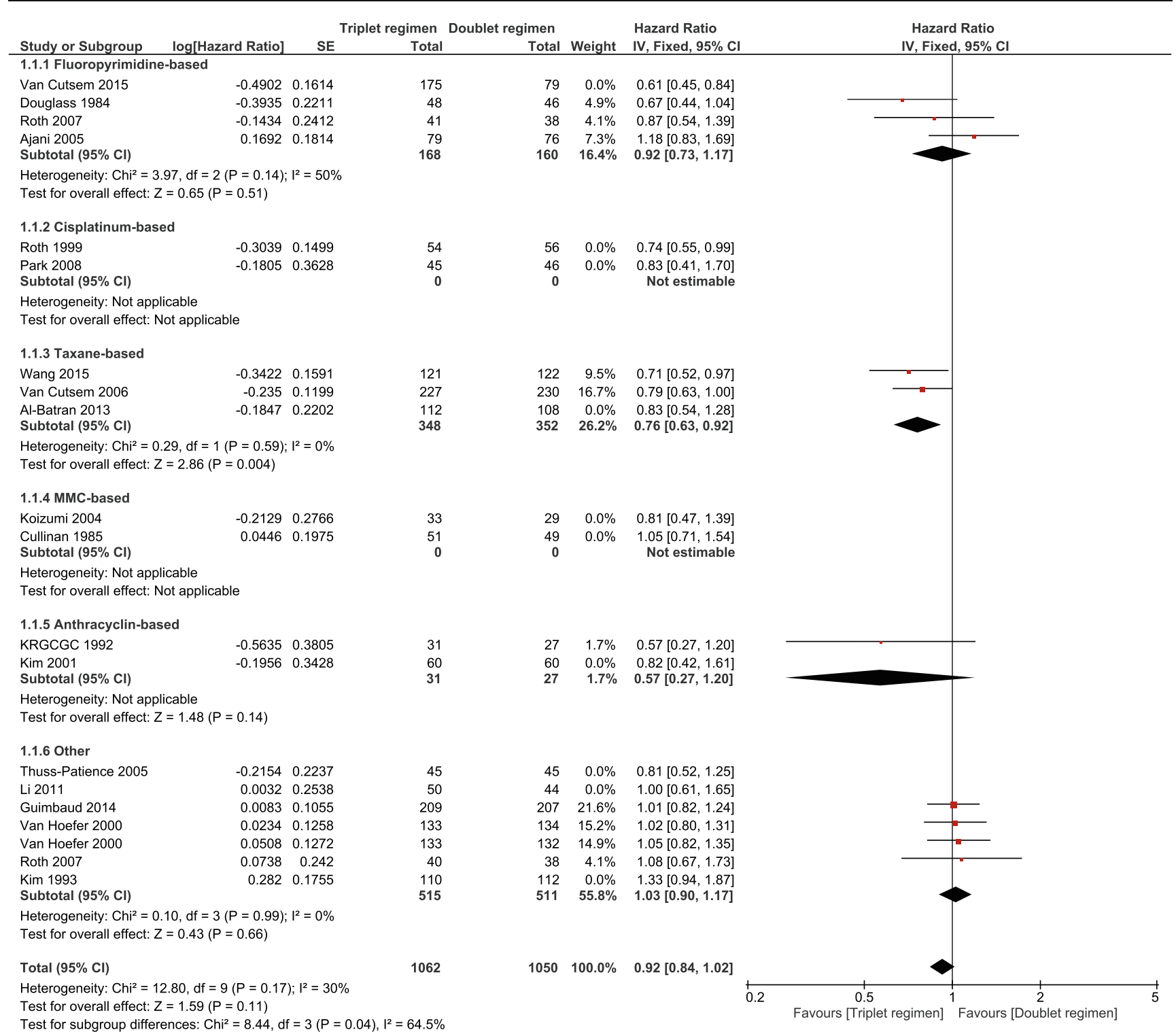

Fig. 7 Sensitivity analysis excluding studies with "unknown" risk of bias on "random sequence" and "allocation concealment"

This contrasts with our finding that the addition of an anthracycline to a doublet did not meet statistical significance for OS. Although the addition of anthracycline to the doublet showed an HR of 0.7 , this was not significant and the number of included patients did not reach 200 in total. It should be noted that Wagner et al. reported a significant HR of 0.77 (95\% CI 0.62-0.95) for the addition of an anthracycline. This was based on three randomized trials, of which the largest study (81.7\% weight) investigated the comparison of an anthracycline-based triplet with a non-anthracyclinebased triplet and showed a significant benefit for the anthracycline-based triplet. The other two included studies were small and compared a triplet versus a doublet but did not show significance. Based on Wagner's data, it may be concluded that an anthracycline-based triplet is superior to a non-anthracycline-based triplet, but based on our results, the true relevance of the addition of anthracyclines to a doublet is doubtful.

In previous meta-analyses, results of PFS and ORR have not been reported. We showed a significant improvement of the secondary endpoint PFS and ORR in favor of triplet chemotherapy, more specifically in the triplets adding a taxane and a taxane or fluoropyrimidine to the combination, respectively. Although in general in metastatic disease ORR is not considered to be the most robust outcome measure, in advanced esophagogastric cancer, ORR may be a clinically relevant end point, given the high symptom burden that patients may suffer from that may be alleviated by response to treatment [1]. 


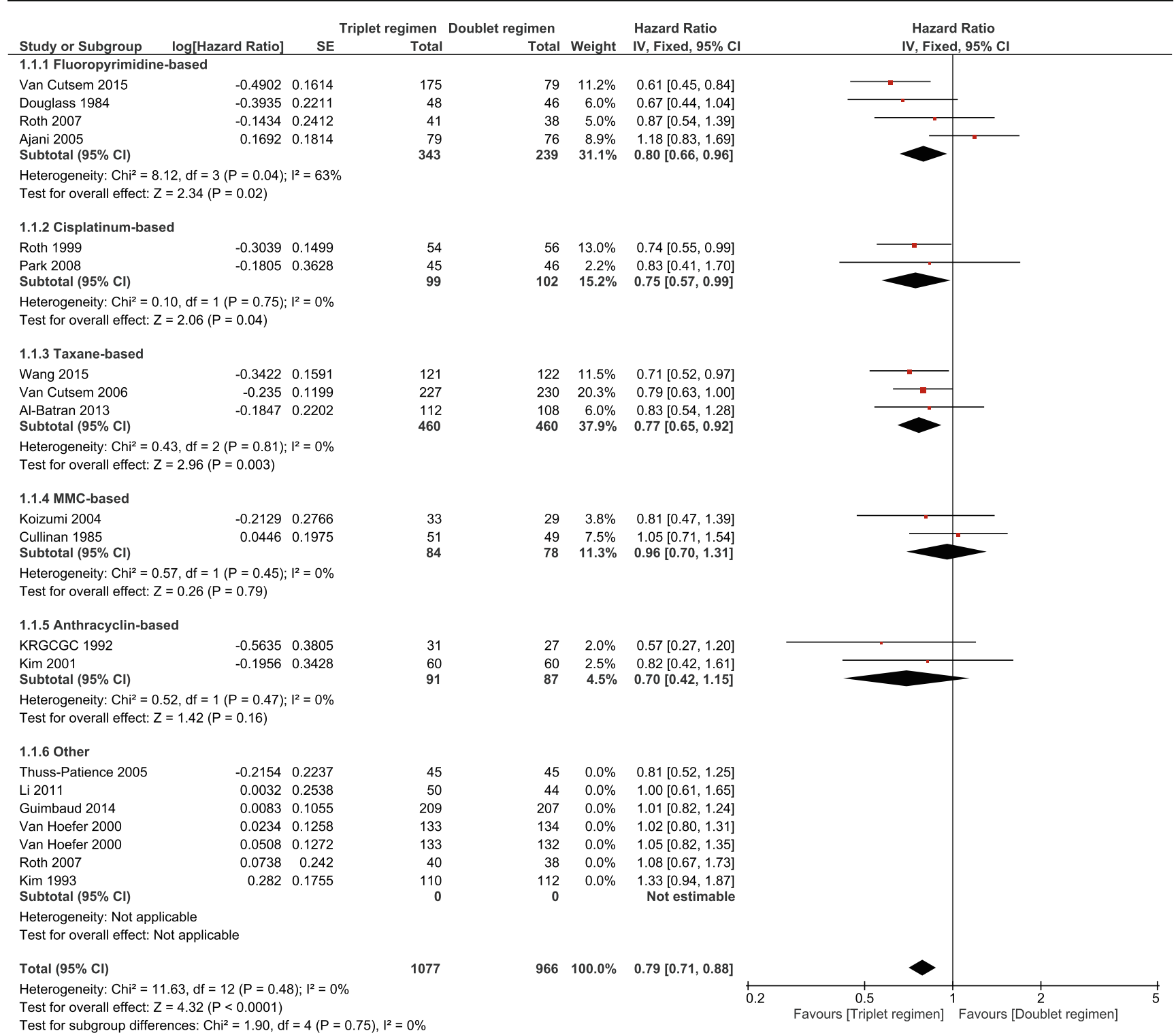

Fig. 8 Sensitivity analysis excluding those trials that compared a triplet versus a doublet without the presence of two identical compounds in both arms

Substantial regional differences have been reported between Asian and non-Asian populations in presentation and subtypes of esophagogastric cancer. For example, in several Asian countries, patients present with early disease, whereas in Western countries the majority of patients present with advanced or metastatic disease [24]. Secondly, while intestinal type of gastric cancer is more prevalent in Asian countries, diffuse gastric cancer is more common in non-Asian countries [25]. Nevertheless, a sensitivity analysis excluding the trials conducted in Asia did not change our results. The sensitivity analysis on the basis of quality of studies in which we excluded the studies with unknown risk of bias may be very firm and can give an underestimation of the quality of the excluded studies. As the findings are consistent with those from the primary analysis, the credibility of our results is strengthened.

Finally, the sensitivity analysis excluding the trials that did not compare at least two identical compounds in both arms did not cause a relevant change of the resulting HR with reduced heterogeneity compared to the original analysis. Hence, the results of this comparison can be considered to be highly robust.

In general, toxicity was significantly higher in the triplet combinations compared to the doublet. In the context of palliative treatment, the degree of toxicity is an important aspect that should be taken into account. Hematologic toxicity has been shown to result in substantial psychological and economic burden [26]. However, others have observed that in patients with 
Table 2 Toxicity grade 3 or 4

\begin{tabular}{|c|c|c|c|c|c|c|c|c|}
\hline \multirow[t]{2}{*}{ Toxicity grade 3 or 4} & \multicolumn{4}{|c|}{ Triplet } & \multicolumn{4}{|c|}{ Doublet } \\
\hline & $N$ & Total & $\%$ & $N$ & Total & $\%$ & $\mathrm{RR}$ & $95 \% \mathrm{CI}$ \\
\hline \multicolumn{9}{|l|}{ Hematologic toxicity } \\
\hline Anemia & 106 & 840 & 12.6 & 121 & 823 & 14.7 & 0.86 & $0.68-1.09$ \\
\hline Neutropenia & 543 & 1006 & 54.0 & 470 & 986 & 47.7 & 1.07 & $0.92-1.23$ \\
\hline Neutropenic fever & 46 & 385 & 11.9 & 46 & 367 & 12.5 & 0.95 & $0.50-1.82$ \\
\hline Thrombocytopenia & 61 & 986 & 6.2 & 37 & 962 & 3.8 & $1.57^{\mathrm{a}}$ & $1.06-2.31$ \\
\hline \multicolumn{9}{|l|}{ Non-hematologic toxicity } \\
\hline Fatigue & 50 & 331 & 15.1 & 52 & 316 & 16.5 & 0.91 & $0.64-1.29$ \\
\hline Infection & 64 & 630 & 10.2 & 40 & 629 & 6.4 & $1.60^{\mathrm{a}}$ & $1.09-2.33$ \\
\hline Mucositis & 59 & 607 & 9.7 & 28 & 591 & 4.7 & $2.20^{\mathrm{a}}$ & $1.00-4.86$ \\
\hline Nausea & 85 & 665 & 12.8 & 63 & 648 & 9.7 & 1.34 & $0.98-1.82$ \\
\hline Vomiting & 84 & 728 & 11.5 & 81 & 716 & 11.3 & 1.04 & $0.78-1.38$ \\
\hline Diarrhea & 114 & 1260 & 9.0 & 98 & 1244 & 7.9 & 0.98 & $0.60-1.61$ \\
\hline Toxicity-related deaths & 68 & 1069 & 6.4 & 54 & 1052 & 5.1 & 1.24 & $0.89-1.74$ \\
\hline
\end{tabular}

$R R$ relative risk, $95 \% C I 95 \%$ confidence interval

${ }^{\text {a }}$ Significant

advanced esophagogastric cancer, toxicity did not directly affect quality of life [1]. Similarly, in a review in colorectal cancer, no correlation was noted between toxicity and quality of life [27].

Although the survival of patients treated with a triplet significantly outweighed the survival of patients treated with a doublet, overall, the survival gain was modest with a hazard ratio of 0.90 of which the clinical relevance may be questioned [28]. However, the benefits of specific triplets, such as fluoropyrimidine, cisplatin, or taxane-based triplets, were more convincing. It should be noted that in this analysis, the effect of second-line treatment has not been taken into consideration. Since second-line treatment has become an accepted standard of care [29-32], adding a third compound in the first line may have become less relevant and could be reserved for second-line or even third-line treatment. Indeed, sequential chemotherapy has been shown equally effective compared to combination-therapy patients with advanced colorectal cancer [33]. However, in contrast to colorectal cancer, in advanced esophagogastric cancer, after progression a substantial part of patients may not be sufficiently fit to undergo second-line treatment $[4,9,34]$. This underscores the relevance of clinical studies incorporating new cytotoxic agents into a triplet, which may be less toxic than currently used agents. For example, the currently recruiting ACTION trial assesses the feasibility of adding the new taxane nab-paclitaxel to the combination of capecitabine and oxaliplatin (clinicaltrials.gov NCT02273713).

Several strengths and limitations of this meta-analysis should be acknowledged. First, the findings regarding OS were very robust, demonstrated by the series of sensitivity analyses. Second, we defined subgroups on the basis of the third chemotherapy compound in order to examine the effect of the adding of a specific compound. However, caution is needed, when interpreting the beneficial effect of triplet versus doublet in terms of PFS because PFS was a secondary outcome in only a limited amount of studies. Moreover, the majority of included studies were phase II studies. Missing information regarding the PFS may under- or overestimate the gain in PFS. Second, toxicity was not uniformly scored which precluded extensive analysis. Third, quality of life was not a designated secondary outcome in most studies, and consequently, no recommendations could be made in this respect. Fourth, not all relevant sensitivity analyses could be conducted as planned, as only $28 \%$ of the authors provided information to evaluate the risk of bias. Fifth, our meta-analysis is not based on individual patient data. Therefore, differences in individual baseline characteristics cannot be adjusted for.

In conclusion, addition of a fluoropyrimidine, cisplatin, or taxane to a doublet showed superior overall survival in first-line treatment of advanced esophagogastric cancer, at the cost, however, of higher toxicity. There is a need for new triplets with cytotoxic agents, which may be less toxic than the currently used regimens.

Conflict of interest The authors declare they have no competing interests.

Open Access This article is distributed under the terms of the Creative Commons Attribution 4.0 International License (http:// creativecommons.org/licenses/by/4.0/), which permits unrestricted use, distribution, and reproduction in any medium, provided you give appropriate credit to the original author(s) and the source, provide a link to the Creative Commons license, and indicate if changes were made. 


\section{References}

1. Al-Batran, S. E., \& Ajani, J. A. (2010). Impact of chemotherapy on quality of life in patients with metastatic esophagogastric cancer. Cancer, 116(11), 2511-8.

2. Murad, A. M., et al. (1993). Modified therapy with 5-fluorouracil, doxorubicin, and methotrexate in advanced gastric cancer. Cancer, 72(1), 37-41.

3. Pyrhonen, S., et al. (1995). Randomised comparison of fluorouracil, epidoxorubicin and methotrexate (FEMTX) plus supportive care with supportive care alone in patients with non-resectable gastric cancer. British Journal of Cancer, 71(3), 587-91.

4. Koizumi, W., et al. (2008). S-1 plus cisplatin versus S-1 alone for first-line treatment of advanced gastric cancer (SPIRITS trial): a phase III trial. Lancet Oncology, 9(3), 215-21.

5. Levi, J. A., et al. (1986). Analysis of a prospectively randomized comparison of doxorubicin versus 5-fluorouracil, doxorubicin, and BCNU in advanced gastric cancer: implications for future studies. Journal of Clinical Oncology, 4(9), 1348-55.

6. Wagner, A. D., et al. (2010). Chemotherapy for advanced gastric cancer. Cochrane Database of Systematic Reviews, 3, CD004064.

7. J. A. and D. A. T. et. al. (2015). National Comprehensive Cancer Network (NCCN) Clinical Practice Guidelines in Oncology, Gastric Cancer. version 1: Available from: http://www.ncen.org/ professionals/physician_gls/pdf/gastric.pdf.

8. Waddell, T., et al. (2013). Gastric cancer: ESMO-ESSO-ESTRO Clinical Practice Guidelines for diagnosis, treatment and follow-up. Annals of Oncology, 24(Suppl 6), vi57-vi63.

9. Bang, Y. J., et al. (2010). Trastuzumab in combination with chemotherapy versus chemotherapy alone for treatment of HER2-positive advanced gastric or gastro-oesophageal junction cancer (ToGA): a phase 3, open-label, randomised controlled trial. Lancet, 376(9742), 687-97.

10. Koizumi, W., et al. (2013). Randomised phase II study of S-1/cisplatin plus TSU-68 vs S-1/cisplatin in patients with advanced gastric cancer. British Journal of Cancer, 109(8), 2079-86.

11. Ohtsu, A., et al. (2011). Bevacizumab in combination with chemotherapy as first-line therapy in advanced gastric cancer: a randomized, double-blind, placebo-controlled phase III study. Journal of Clinical Oncology, 29(30), 3968-76.

12. Cunningham D. et al., (2013). RILOMET-1: an international phase III multicenter, randomized, double-blind, placebo-controlled trial of rilotumumab plus epirubicin, cisplatin, and capecitabine (ECX) as first-line therapy in patients with advanced ME-positive gastric or gastroesophageal junction (G/GEJ) adenocarcinoma. Journal of Clinical Oncology, 31 (Suppl;abstr TPS4153).

13. Higgins, J.P. and S. Green. (2011). Cochrane Handbook for Systematic Reviews of Interventions.

14. Tierney, J. F., et al. (2007). Practical methods for incorporating summary time-to-event data into meta-analysis. Trials, 8, 16.

15. Higgins, J. P., \& Thompson, S. G. (2002). Quantifying heterogeneity in a meta-analysis. Statistics in Medicine, 21(11), 1539-58.

16. Ajani, J. A., et al. (2005). Phase II multi-institutional randomized trial of docetaxel plus cisplatin with or without fluorouracil in patients with untreated, advanced gastric, or gastroesophageal adenocarcinoma. Journal of Clinical Oncology, 23(24), 5660-7.

17. Al-Batran, S. E., et al. (2013). The feasibility of triple-drug chemotherapy combination in older adult patients with oesophagogastric cancer: a randomised trial of the Arbeitsgemeinschaft Internistische Onkologie (FLOT65+). European Journal of Cancer, 49(4), 83542.

18. Guimbaud, R., et al. (2014). Prospective, randomized, multicenter, phase III study of fluorouracil, leucovorin, and irinotecan versus epirubicin, cisplatin, and capecitabine in advanced gastric adenocarcinoma: a French intergroup (Federation Francophone de
Cancerologie Digestive, Federation Nationale des Centres de Lutte Contre le Cancer, and Groupe Cooperateur Multidisciplinaire en Oncologie) study. Journal of Clinical Oncology, 32(31), 3520-6.

19. Park, S. H., et al. (2008). Randomized phase II study of irinotecan, leucovorin and 5-fluorouracil (ILF) versus cisplatin plus ILF (PILF) combination chemotherapy for advanced gastric cancer. Annals of Oncology, 19(4), 729-33.

20. Roth, A. D., et al. (2007). Docetaxel, cisplatin, and fluorouracil; docetaxel and cisplatin; and epirubicin, cisplatin, and fluorouracil as systemic treatment for advanced gastric carcinoma: a randomized phase II trial of the Swiss Group for Clinical Cancer Research. Journal of Clinical Oncology, 25(22), 3217-23.

21. Van Cutsem, E., et al. (2015). Docetaxel plus oxaliplatin with or without fluorouracil or capecitabine in metastatic or locally recurrent gastric cancer: a randomized phase II study. Annals of Oncology, 26(1), 149-56.

22. Van Cutsem, E., et al. (2006). Phase III study of docetaxel and cisplatin plus fluorouracil compared with cisplatin and fluorouracil as first-line therapy for advanced gastric cancer: a report of the V325 Study Group. Journal of Clinical Oncology, 24(31), 4991-7.

23. Vanhoefer, U., et al. (2000). Final results of a randomized phase III trial of sequential high-dose methotrexate, fluorouracil, and doxorubicin versus etoposide, leucovorin, and fluorouracil versus infusional fluorouracil and cisplatin in advanced gastric cancer: a trial of the European Organization for Research and Treatment of Cancer Gastrointestinal Tract Cancer Cooperative Group. Journal of Clinical Oncology, 18(14), 2648-57.

24. Ohtsu, A., Yoshida, S., \& Saijo, N. (2006). Disparities in gastric cancer chemotherapy between the East and West. Journal of Clinical Oncology, 24(14), 2188-96.

25. Crew, K. D., \& Neugut, A. I. (2006). Epidemiology of gastric cancer. World Journal of Gastroenterology, 12(3), 354-62.

26. Liou, S. Y., et al. (2007). Economic burden of haematological adverse effects in cancer patients: a systematic review. Clinical Drug Investigation, 27(6), 381-96.

27. Funaioli, C., Longobardi, C., \& Martoni, A. A. (2008). The impact of chemotherapy on overall survival and quality of life of patients with metastatic colorectal cancer: a review of phase III trials. Journal of Chemotherapy, 20(1), 14-27.

28. Willemse P. H., C. J. Rodenburg, and O. R. (2001). [Een toetssteen voor besluitvorming: nieuwe criteria voor regsistraie oncologische middelen]. Medisch Contact, 56(20): p. 794-6.

29. Ford, H. E., et al. (2014). Docetaxel versus active symptom control for refractory oesophagogastric adenocarcinoma (COUGAR-02): an open-label, phase 3 randomised controlled trial. Lancet Oncology, 15(1), 78-86.

30. Kim, H. S., et al. (2013). Second-line chemotherapy versus supportive cancer treatment in advanced gastric cancer: a meta-analysis. Annals of Oncology, 24(11), 2850-4.

31. Thuss-Patience, P. C., et al. (2011). Survival advantage for irinotecan versus best supportive care as second-line chemotherapy in gastric cancer-a randomised phase III study of the Arbeitsgemeinschaft Internistische Onkologie (AIO). European Journal of Cancer, 47(15), 2306-14.

32. Wilke, H., et al. (2014). Ramucirumab plus paclitaxel versus placebo plus paclitaxel in patients with previously treated advanced gastric or gastro-oesophageal junction adenocarcinoma (RAINBOW): a double-blind, randomised phase 3 trial. Lancet Oncology, 15(11), 1224-35.

33. Koopman, M., et al. (2007). Sequential versus combination chemotherapy with capecitabine, irinotecan, and oxaliplatin in advanced colorectal cancer (CAIRO): a phase III randomised controlled trial. Lancet, 370(9582), 135-42. 
34. Cunningham, D., et al. (2008). Capecitabine and oxaliplatin for advanced esophagogastric cancer. New England Journal of Medicine, 358(1), 36-46.

35. Cullinan, S. A., et al. (1985). A comparison of three chemotherapeutic regimens in the treatment of advanced pancreatic and gastric carcinoma. Fluorouracil vs fluorouracil and doxorubicin vs fluorouracil, doxorubicin, and mitomycin. JAMA, 253(14), 2061-7.

36. Douglass, H. O., Jr., et al. (1984). An Eastern Cooperative Oncology Group evaluation of combinations of methyl-CCNU, mitomycin $\mathrm{C}$, adriamycin, and 5-fluorouracil in advanced measurable gastric cancer (EST 2277). Journal of Clinical Oncology, 2(12), 1372-81.

37. Kim, N. K., et al. (1993). A phase III randomized study of 5fluorouracil and cisplatin versus 5-fluorouracil, doxorubicin, and mitomycin $\mathrm{C}$ versus 5-fluorouracil alone in the treatment of advanced gastric cancer. Cancer, 71(12), 3813-8.

38. Kim T., Choi S. J., and e.a. Ahn J. H. (2001). A prospective randomized phase III trial of 5-fluorouracil and cisplatin (FP) versus epirubicin, cisplatin, and $5 F U(E C F)$ in the treatment of patients with previously untreated advanced gastric cancer (AGC). Eur J Cancer 37 (Suppl 6;S314).

39. Koizumi, W., et al. (2004). Randomized phase II study comparing mitomycin, cisplatin plus doxifluridine with cisplatin plus doxifluridine in advanced unresectable gastric cancer. Anticancer Research, 24(4), 2465-70.

40. Kyoto Research Group for Chemotherapy of Gastric Cancer (KRGCGC). (1992). A randomized, comparative study of combination chemotherapies in advanced gastric cancer: 5-fluorouracil and cisplatin (FP) versus 5-fluorouracil, cisplatin, and 4'-epirubicin (FPEPIR). Anticancer Research, 12(6B), 1983-8.

41. Li, X. D., et al. (2011). Paclitaxel based vs oxaliplatin based regimens for advanced gastric cancer. World Journal of Gastroenterology, 17(8), 1082-7.

42. Lin R, et al.,.(2009). Phase IIb trial of fluorouracil, leucovorin, oxaliplatin, and paclitaxel (POF) compared with fluorouracil, leucovorin, and irinotecan (IF) as first-line treatment for advanced gastric cancer (AGC). J Clin Oncol 27 (suppl;abstr e 15642).

43. Maiello E, et al.,(2011). Epirubicin (E) in combination with cisplatin $(C D D P)$ and capecitabine $(C)$ versus docetaxel $(D)$ combined with 5-fluorouracil $(\%-F U)$ by continuous infusion as front-line therapy in patients with advanced gastric cancer (AGC): preliminary results of a randomized. 29 (4 suppl; abstr 97).

44. Roth, A., et al. (1999). High doses of 5-fluorouracil and epirubicin with or without cisplatin in advanced gastric cancer: a randomized study. Tumori, 85(4), 234-8.

45. Thuss-Patience, P. C., et al. (2005). Docetaxel and continuousinfusion fluorouracil versus epirubicin, cisplatin, and fluorouracil for advanced gastric adenocarcinoma: a randomized phase II study. Journal of Clinical Oncology, 23(3), 494-501.

46. Wang, J., et al.,(2015). Randomized multicenter phase III study of a modified docetaxel and cisplatin plus fluorouracil regimen compared with cisplatin and fluorouracil as first-line therapy for advanced or locally recurrent gastric cancer. Gastric Cancer.

47. Yun, J., et al. (2010). A randomised phase II study of combination chemotherapy with epirubicin, cisplatin and capecitabine (ECX) or cisplatin and capecitabine (CX) in advanced gastric cancer. European Journal of Cancer, 46(5), 885-91. 